\title{
A web application for solving puzzles with logic grids: design, user tests, and first experiences in a Logic course
}

\author{
Leonel Morales Díaz, Lissette Fleck de Umaña
}

Published: 30 November 2021

\begin{abstract}
Searching for new in-class activities for a Symbolic Logic course, we found a type of puzzle that uses a specially constructed matrix for solving. They were available on websites in English and possibly in other languages, but not in Spanish. Their interactive nature and logical structure make them appropriate for the class although it was clear that Spanish versions of the puzzles would be needed. Puzzle statements presented in websites in English appeared ungenuine or artificial, and often out of context. In this article, we present a description of this type of logic puzzle, the problems found when using them in websites in English, the process followed to design and create a web application for distribution and solving puzzles in Spanish, and the user tests conducted. The prototype presented here includes modifications to the user interface, internal algorithms, and the process to create the enunciates and clues of the puzzle.
\end{abstract}

\section{Keywords:}

Logic; Puzzles; User-Centered Design; Games; Web Applications; Education.

\section{Introducción}

Las matrices lógicas, logic grids en Inglés, son una herramienta de resolución de problemas empleada cuando se necesita encontrar combinaciones únicas y mutuamente excluyentes de valores en diferentes categorías. Se aplican a problemas cerrados, es decir, de solución restringida a un conjunto bien definido de posibilidades. Al plantearlos se presenta un conjunto de categorías y cantidades iguales de valores en cada una. También se ofrecen pistas que ayudan a encontrar las combinaciones que solucionan el problema. Con el uso de las pistas en el planteamiento, se invita al razonamiento y la aplicación de reglas lógicas, pero de una forma entretenida y divertida. Por ello, se les puede llamar acertijos

Morales Díaz, Leonel V., Fleck de Umaña, Lissette.

Universidad Francisco Marroquín

Guatemala, Guatemala.

litomd@ufm.edu, afleck@ufm.edu lógicos. La Figura 1 presenta un ejemplo de este tipo de problemas y la matriz lógica que ayuda a solucionarlo.
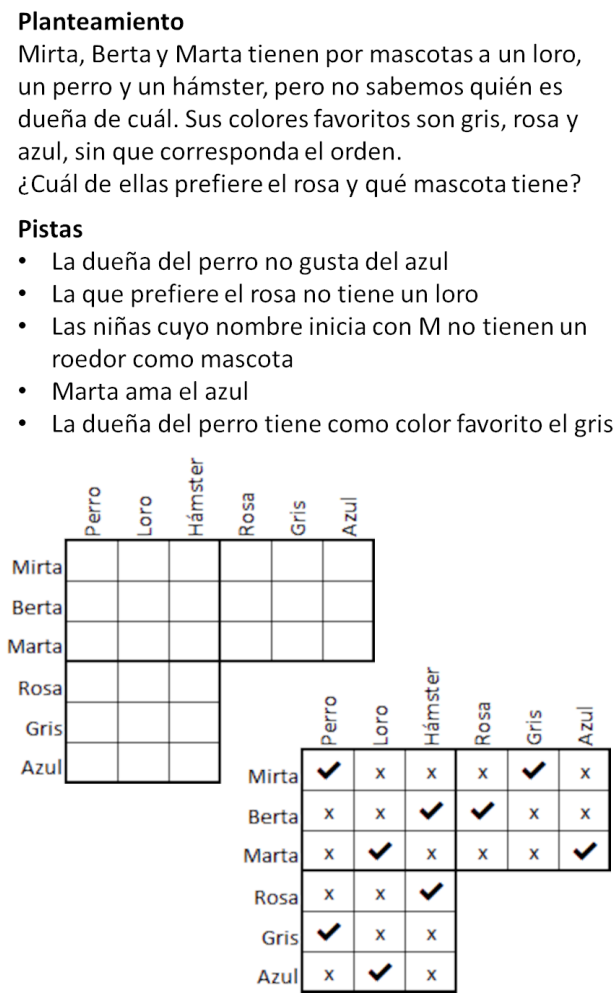

Figura 1. Ejemplo de un acertijo lógico con planteamiento, pistas, matriz lógica para resolver y matriz resuelta. Disponible en http://litomd.com/logicgrid/?game=1000.

Otras descripciones de esta herramienta pueden encontrarse en [4] que además presenta el sistema de resolución automatizada llamado LOGICIA, y en [8] y [9] que son sitios web dedicados a estos problemas. El enfoque de este trabajo es diferente porque no se pretende la solución automatizada, sino el ejercicio mental humano y porque los enunciados y pistas se presentan, por primera vez, en Español. De hecho, en el transcurso de la investigación presentada en este artículo, no fue posible encontrar otros ejemplos de acertijos lógicos de este estilo en Español. 
Varios autores latinoamericanos han estudiado el uso de juegos como herramientas didácticas para enseñar lógica. En [6] los autores presentan una propuesta de juego serio para fortalecer el razonamiento lógico-matemático y evalúan su eficacia. El uso de juegos populares para el desarrollo del pensamiento lógicomatemático fue el enfoque utilizado en [5], empleando encuestas cómo método de validación. Una variación interesante se presenta en [2] que primero observa, estudia, y entrevista a un grupo de estudiantes de capacidades excepcionales y a sus docentes, y entonces procede a diseñar juegos para ellos. Sin embargo, ninguno explora el uso de matrices lógicas o acertijos del tipo descrito aquí.

El proyecto que ahora presentamos se enmarca en uno de mayor alcance, que busca determinar si la utilización de acertijos lógicos, del tipo que puede resolverse con matrices lógicas, presenta algún beneficio para el desarrollo de las habilidades de análisis y razonamiento lógico, y si su utilización como herramienta auxiliar didáctica mejora la experiencia de los participantes en cursos relacionados con la lógica como el descrito en [11]. Estos objetivos no pueden buscarse ni conseguirse sin un soporte práctico: un conjunto de acertijos lógicos con sus pistas, con la cantidad correcta de valores por cada categoría, una solución única que combina todos esos valores, y una forma práctica de distribuirlos y resolverlos, en formato digital o en papel.

Conseguir ese soporte mediante el diseño de una aplicación web, por los problemas que presentan el papel o la pizarra, y plantear un método para crear nuevos acertijos, es la propuesta concreta en esta etapa del proyecto. Buscamos aplicar principios de diseño centrado en el usuario [10] para crear una aplicación fácil de usar, prestando atención a toda la experiencia interactiva [7].

\section{Problemas Identificados}

Este proyecto se originó por la necesidad de ofrecer a los estudiantes de un curso de lógica una alternativa divertida para la práctica de los conceptos presentados en clase. Al principio los acertijos se tomaban directamente de sitios web como [9] y se resolvían en el aula. Se identificaron dos problemas con este enfoque: 1. Los acertijos están planteados en Inglés con historias y pistas que resultan descontextualizadas $y$, en ocasiones, poco creíbles. 2. Los sitios web tienen un diseño con publicidad e invitaciones a visitar otras páginas o secciones del sitio, lo que resulta en distracciones y desenfoque.

Una primera alternativa fue usar acertijos lógicos ideados por los profesores del curso. Al plantearlos en clase, dibujar la matriz lógica se hizo parte del trabajo, lo cual tiene aspectos positivos ya que implica analizar con cuidado el problema y ser ordenado para colocar correctamente cada serie de valores, pero toma tiempo y distrae del contenido central del problema.

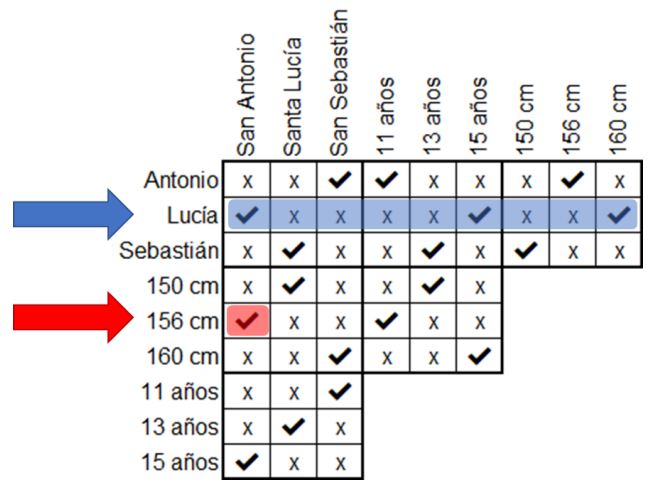

Figura 2. Ejemplo de matriz lógica con solución incongruente. Disponible en http://litomd.com/logicgrid/?game=1002.
Otra estrategia fue la de asignar a equipos de alumnos la tarea de crear un acertijo lógico con planteamiento y pistas, dibujar la matriz lógica, y resolverlo en clase con la participación de sus compañeros. En general funcionó bien pero el hecho de que se trataba de problemas inventados en ocasiones los hacía parecer forzados o poco creíbles.

En este punto identificamos otro problema. La combinación de valores de categorías debe ser congruente, o bien, para usar un término técnico, debe cumplir con la cerradura triádica o la transitividad de relaciones. Si la combinación $a-b$ es parte de la solución y b-c también, entonces c-a debe ser parte de la solución o hay un error. La Figura 2 muestra un ejemplo. La fila de Lucía indica que ella vive en San Antonio, tiene 15 años y mide $160 \mathrm{~cm}$, pero la columna San Antonio indica que ahí vive Lucía que mide $156 \mathrm{~cm}$, lo cual es incongruente. La cerradura triádica requiere que si los pares (Lucía, San Antonio) y (Lucía, $160 \mathrm{~cm}$ ) pertenecen a la solución, entonces el par (San Antonio, $160 \mathrm{~cm}$ ) también debe ser parte de ella. Es difícil verificar esta condición cuando se trabaja en papel o en el pizarrón. Fue sorprendente encontrar que los sitios web de acertijos lógicos tampoco toman esto en cuenta.

Adicionalmente, fue notorio que algunos estudiantes se involucraron más en resolver el acertijo y otros simplemente observaban o perdían interés. Cuando el acertijo se resolvía con una matriz lógica en el pizarrón con las pistas a la vista, alguien podría llamar la atención sobre una pista y sus implicaciones, interrumpiendo el proceso de análisis que otro podía estar haciendo.

En resumen, los problemas identificados son los siguientes: a) acertijos en Inglés con historias descontextualizadas y poco creíbles; b) distracción causada por sitios web con publicidad $u$ otros elementos visuales; c) dibujar la matriz lógica distrae, consume tiempo y es fácil equivocarse; d) los acertijos con historias y pistas inventadas pueden parecer forzados; e) es difícil verificar que se cumpla con la cerradura triádica en la solución.

\section{Proceso de Diseño}

Con la experiencia y observaciones realizadas, el primer paso de diseño fue plantear historietas (storyboards) para ilustrar la experiencia que buscábamos proporcionar a los usuarios con la aplicación web independientemente del dispositivo. Se plantearon dos escenarios: el primero comprende usuarios que encuentran la aplicación por referencia de alguien o por sus recorridos en Internet; el segundo se dedica específicamente a alumnos de un curso de lógica. El resultado se resume así: los usuarios independientes deben percibir la aplicación como divertida y una buena ocasión para ejercitar la mente; los que la empleen en el contexto de un curso deben apreciar la facilidad que les brinda para seguir una actividad en clase, haciéndolos sentir que están participando, y que comprenden mejor de lo que se trata.

En este punto el trabajo se concentró en encontrar un método para generar acertijos lógicos del tipo que puede resolverse con una matriz lógica. La solución fue crearlos a partir de personajes históricos tomados en grupos de 4, por ejemplo, matemáticos de la Antigua Grecia, filósofos ilustrados del siglo XVIII, libertadores de América, ganadores de premios Nobel de una disciplina o época concreta, y otras variaciones. Para cada grupo se identifica alguna peculiaridad como el año o lugar de nacimiento, su obra principal, u otra que se preste como categoría del acertijo. También exploramos temas de geografía, tomando grupos de municipios, lagos, volcanes, ríos o estados. Las civilizaciones prehispánicas, los mayores fabricantes de automóviles del mundo, participantes en juegos olímpicos, planetas del sistema solar, entre otros, fueron temas de alguno de los acertijos que diseñamos. 
Utilizar referencias de las materias mencionadas permite revestir al acertijo de credibilidad. Aparentemente, resolver estos problemas refuerza y reaviva conocimientos previos pues invita a relacionarlos de una manera diferente: quién nació primero, qué lago es más grande, qué ciudad maya entró en conflicto con otra, son consideraciones que habitualmente no se hacen cuando se estudia cada personaje o accidente geográfico. Estudiar los beneficios que esto pueda traer excede el alcance de esta investigación. Por otra parte, por tratarse de hechos establecidos, estos acertijos podrían resolverse sin analizar las pistas, recurriendo a búsquedas en Internet. Sin embargo, hemos visto que esto no resulta práctico ya que requiere integrar información dispersa. Nuevamente, confirmar esto excede los límites del proyecto. Por ahora nos conformamos con indicar a quien los resuelve, que no es buena idea buscar la solución en Internet.

Al contar con el contenido del acertijo, se debe configurar adecuadamente la matriz lógica para resolverlo. La Figura 3 muestra cómo hacerlo. Algunas versiones de estos problemas fueron incluidas en exámenes y ejercicios para los alumnos del curso de lógica y en otros materiales educativos. Estos ensayos en papel proporcionaron retroalimentación. Resultó interesante observar que se podían conseguir diferentes grados de dificultad.

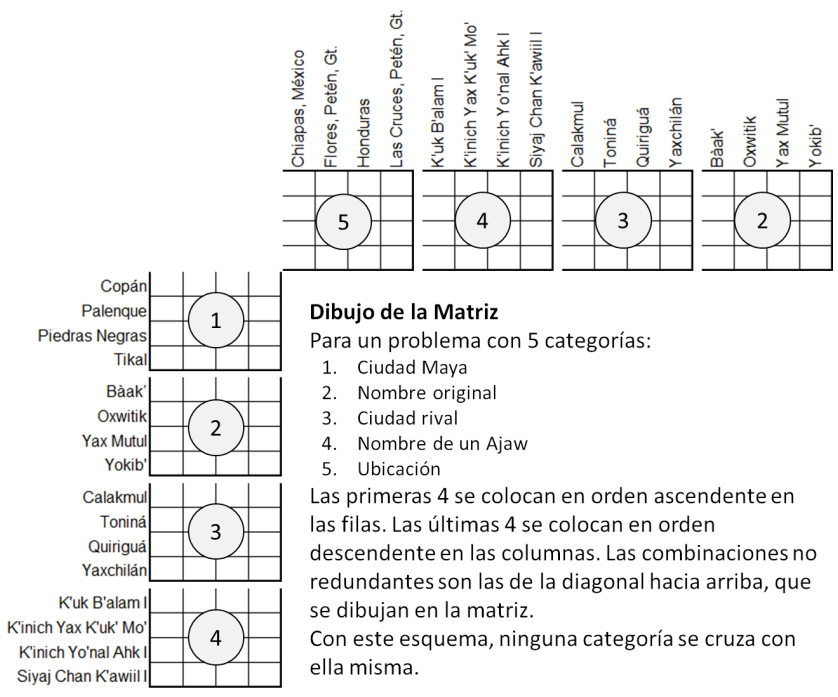

Figura 3. Proceso de dibujo de matriz lógica. Disponible en http://litomd.com/logicgrid/?game=9000.

El primer prototipo digital interactivo creado, sirvió para afinar al algoritmo de dibujo de la matriz y las respuestas a las interacciones de los usuarios usando el mismo esquema simple que se usa en sitios web como [9]. En él, el primer clic coloca una equis en la casilla para indicar que esa combinación no es parte de la solución; el segundo clic cambia de equis a cheque $(\checkmark$ código Unicode 2713 [12] aunque se pueden usar otros símbolos) indicando que esa combinación es parte de la solución y además anula con equis el resto de casillas en la misma fila y columna; un tercer clic borra el cheque y regresa las casillas en la misma fila y columna, a su estado anterior, lo cual implica que la aplicación debe mantener cierta memoria de los estados previos.

Para el prototipo se utilizó la plataforma de programación en Khan Academy que permite utilizar JavaScript y la biblioteca ProcessingJS [13]. Al disponer de un prototipo interactivo robusto, el código fue migrado a una página para convertirlo en aplicación web. Con ello ya fue posible unir los dos componentes diseñados: los acertijos lógicos creados anteriormente y la aplicación web que facilita su distribución hacia cualquier dispositivo que disponga de un navegador suficientemente moderno.

\section{Pruebas con Usuarios}

El objetivo de las pruebas con usuarios fue determinar si se estaba consiguiendo la experiencia de usuario mostrada en las historietas (storyboards) creadas para el efecto, en los dos escenarios: usuarios independientes (Figura 4, izquierda) y alumnos usando la aplicación dentro del aula (Figura 4, derecha).
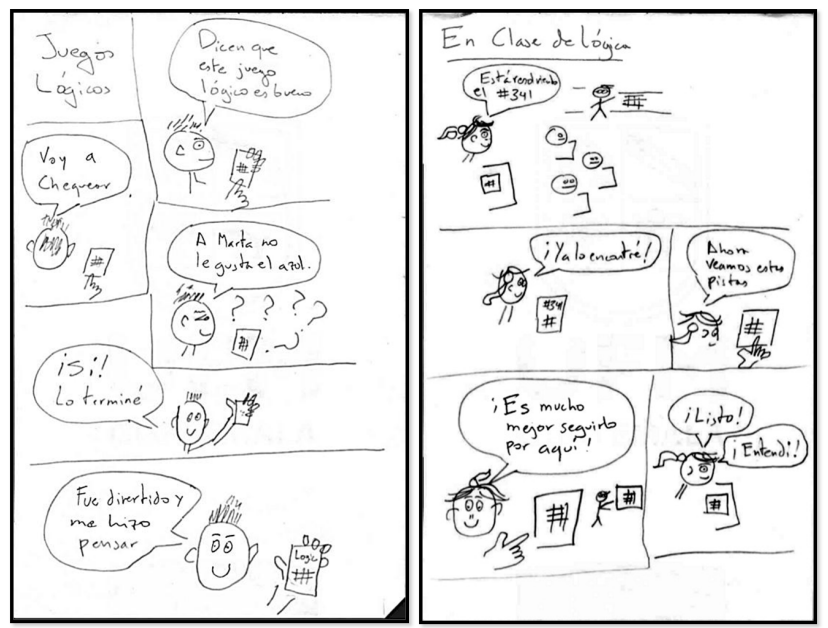

Figura 4. Historietas (storyboards) que ilustran la experiencia esperada en los usuarios independientes (izquierda) y en los alumnos que utilizan la aplicación dentro del aula (derecha).

Se emplearon tres estrategias para obtener retroalimentación. La primera, publicar acertijos lógicos en redes sociales, especialmente Twitter y LinkedIn. Como la aplicación informa el tiempo utilizado para resolver el acertijo junto con una celebración visual, algunos usuarios publicaron capturas de pantalla para mostrar los tiempos logrados. Otros sugirieron temas de acertijos o agradecieron el esfuerzo llegando a llamarlo importante.

La segunda estrategia consistió en pedir a profesores de diferentes materias que resolvieran un acertijo. Esta actividad reveló que la mecánica de la matriz no era intuitiva ni se podía deducir a simple vista. Se añadió al interfaz de usuario una pequeña animación, visible siempre, que muestra cómo trabajar en la matriz. Un profesor comentó que le gustaba resolverlos pero que prefería hacerlo en su computadora portátil porque en el teléfono era difícil ver las pistas y lograr marcar la casilla correcta, posible indicativo del problema en interfaces conocido como dedo gordo (fat finger) descrito en la literatura [1] [3].

La tercera fue recurrir a estudiantes que ya habían tomado cursos de lógica y conocían los acertijos que se resuelven con matrices lógicas por haberlos utilizado en versiones en línea en idioma Inglés y en papel con planteamientos en Español. Se les pidió probar la aplicación web y hacer comentarios. Propusieron mejoras en la adaptación del tamaño de la matriz al espacio disponible en la ventana del navegador y cambiar el fondo de pantalla pues era de un color apagado y se sentía aburrido. Sus sugerencias fueron tomadas en cuenta.

En este punto también se corrigió el problema de la verificación de cerradura triádica. Si la matriz se ha llenado correctamente esta verificación pasa desapercibida, pero si hay alguna incongruencia esta se muestra con un tono de rojo en la matriz y un mensaje para el usuario. 


\section{Uso en el Aula}

La versión disponible se considera un prototipo de alta fidelidad y por lo mismo se ha iniciado su implementación en un curso de lógica. Al momento de escribir este reporte, se han ensayado dos formas de utilización: resolución de acertijo lógico por todo el grupo (más de 30 estudiantes conectados por enlace remoto) y resolución en grupos pequeños. Los alumnos abren el acertijo lógico en la aplicación web y pueden seguir el análisis de las pistas que se hace por todo el grupo o pueden examinarlas individualmente si lo prefieren. Usar la aplicación en lugar de resolver el acertijo en papel o en el pizarrón parece brindar una sensación de seguridad a quien lo está resolviendo, al mismo tiempo, reta e invita a completarlo y "ganar el juego".

Un hallazgo que llamó la atención fue la dificultad de los alumnos para manejar pistas presentadas como condicional, en las que el consecuente es falso y por tanto el antecedente también debe ser falso (Modus Tollens). Con las pistas del problema se plantea el condicional y más adelante se deduce que el consecuente no puede ser verdadero lo cual debiera llevar a negar el antecedente. Esta deducción no resultó evidente para algunos estudiantes.

Otras variantes de uso incluyen la resolución de un acertijo por un alumno ante el resto de la clase, que puede ayudarlo con alguna pista a solicitud del mismo alumno, y la designación de un equipo pequeño de alumnos para realizar esa misma tarea. Lo que se busca con estas modalidades es que quienes tienen a su cargo resolver el problema puedan concentrarse en hacerlo y llevar el hilo conductor del proceso sin las distracciones que se dan cuando todos pueden aportar ideas. La intención es resolver los acertijos para introducir un momento de descanso y reactivación mental a mitad de las clases de cursos de lógica, que en ocasiones pueden resultar agotadoras.

\section{Trabajo Futuro}

Al escribir este artículo la lista de acertijos lógicos listos para usarse tiene cerca de 30 elementos y se agregan 1 o 2 por semana. Esto es suficiente para un semestre del curso. Para facilitar el proceso de creación, se necesita una herramienta de edición que tenga opciones de previsualización y que en este momento aún no existe. Por ahora, los acertijos se guardan como archivos en formato JSON editados manualmente. Una de las partes que más tiempo consume, es idear las pistas y asegurar que son suficientes para resolver el acertijo. Con la herramienta de edición, también será posible reclutar autores que deseen colaborar como creadores de acertijos.

También se necesita un directorio de acertijos que permita seleccionar con facilidad uno para usarlo en clase. La herramienta de edición y el directorio son proyectos a mediano plazo. En el largo plazo será necesario hacer nuevas evaluaciones de usabilidad y experiencia de uso tanto para la aplicación web como para las herramientas auxiliares. Los efectos positivos que pudieran derivarse del uso de la aplicación web en el marco de un curso de lógica deberán evaluarse con cierta periodicidad.

\section{Conclusiones}

En este artículo hemos presentado el proceso de diseño que se siguió para construir y evaluar una aplicación web que permite resolver acertijos lógicos con matrices. La aplicación resuelve el problema de la distribución digital del acertijo y otros que estaban presentes antes de disponer de ella: a) acertijos en Inglés y descontextualizados ahora están redactados en Español y con planteamientos y pistas sobre sucesos y lugares reales; b) distracción causada por sitios web con publicidad, ahora los acertijos se presentan en un diseño limpio que permite enfocarse en encontrar la solución; c) distracción y errores por tener que dibujar la matriz lógica, ahora es un proceso automático y sin errores; d) acertijos inventados con historias y pistas que parecían forzados, ahora se usan hechos históricos o relativos a objetos naturales existentes; e) cerradura triádica era difícil de verificar, ahora se verifica automáticamente dentro de la aplicación.

Utilizando los principios del diseño centrado en el usuario, se probó la aplicación con usuarios representativos desde sus primeros prototipos. Los hallazgos llevaron a mejoras de la aplicación, hasta llevarla a su estado actual que puede considerarse un prototipo de alta fidelidad, que ha empezado a emplearse con resultados alentadores como parte de un curso de lógica.

Siendo este un proyecto que forma parte de otro de mayor alcance, describimos el trabajo por realizar a mediano y largo plazo. Los comentarios recibidos hasta ahora han contribuido a mejorar la aplicación, que constituye la primera herramienta en Español que ofrece matrices lógicas para solucionar acertijos, con contenidos adecuados para el entorno educativo latinoamericano.

\section{Referencias}

[1] Aurora Barrero, David Melendi, Xabiel G. Paneda, Roberto Garcia, Laura Pozueco, and Jose Luis Arciniegas. 2015. A research on typing methods for interactive Digital Television Applications. IEEE Latin America Transactions 13, 11: 3612-3620.

[2] Iván Andrés Padilla Escorcia. 2018. El juego y la inteligencia lógico-matemática de estudiantes con capacidades excepcionales. Educación y humanismo 20, 35: 166-183.

[3] I. Scott MacKenzie. 2012. Human-computer interaction: An empirical research perspective.

[4] Arindam Mitra and Chitta Baral. 2015. Learning to Automatically Solve Logic Grid Puzzles. Proceedings of the 2015 Conference on Empirical Methods in Natural Language Processing, Association for Computational Linguistics, 1023-1033.

[5] Geofre Pinos Morales, Diana Ayala Gavilanes, y Diego Bonilla Jurado. 2018. Desarrollo del pensamiento lógicomatemático a través de juegos populares y tradicionales en niños de educación inicial. Revista Científica Ciencia y tecnología 18, 19.

[6] Alicia López Sánchez y Aída González Lara. 2021. Evaluación de un juego serio que contribuye a fortalecer el razonamiento lógico-matemático en estudiantes de nivel medio superior. RIED. Revista Iberoamericana de Educación a Distancia 24, 1: 221-243.

[7] Sebastián Sastoque, Cristian Narváez, y Germán Garnica. 2016. Metodología para la construcción de Interfaces Gráficas Centradas en el Usuario. Nuevas Ideas en Informática Educativa 12: 314-324.

[8] Logic Puzzles - Aha! Puzzles. Recuperado el 27 de Octubre de 2021 desde https:// www.ahapuzzles.com/logic/logicpuzzles/

[9] Logic Puzzles by Puzzle Baron. Recuperado el 4 de Agosto de 2021 desde https://logic.puzzlebaron.com/.

[10] Leonel Morales Díaz and Laura Gaytán-Lugo. 2016. Sobre Usuarios y Multitudes: Consideraciones Históricas en la Interacción Humano- Computadora. Revista Faz 9: 148-161.

[11] Leonel V. Morales Díaz and Moris A. Polanco. 2011. Using an On-Line Platform to Teach Logic. ICBL 2011: 97. 
[12] Unicode Utilities: Character Properties. Recuperado el 8 de Agosto de 2021 desde

https://util.unicode.org/UnicodeJsps/character.jsp?a=2713.
[13] Aprender programación en Khan Academy (artículo). Khan Academy. Recuperado el 8 de Agosto de 2021 desde https://es.khanacademy.org/computing/computerprogramming/programming/intro-toprogramming/a/learning-programming-on-khan-academy.

\section{(c) (1) (\$) $\Theta$}

(C) 2021 by the authors. This work is licensed under the Creative Commons AttributionNonCommercial-NoDerivatives 4.0 International License. To view a copy of this license, visit http://creativecommons.org/licenses/by-nc-nd/4.0/ or send a letter to Creative Commons, PO Box 1866, Mountain View, CA 94042, USA. 